De Tampere à Séville : bilan de la sécurité européenne $(1 / 2)$

\title{
Le Traité d'Amsterdam : enjeux et défis en matière d'égalité de traitement
}

The Amsterdam Treaty: Issues and Challenges concerning Equal Trea

Isabelle Chopin

\section{(2) OpenEdition}

Journals

Édition électronique

URL : http://journals.openedition.org/conflits/765

DOI : $10.4000 /$ conflits.765

ISSN : 1777-5345

Éditeur :

CCLS - Centre d'études sur les conflits lilberté et sécurité, L'Harmattan

Édition imprimée

Date de publication : 1 mars 2002

Pagination : $19-25$

ISBN : 2-7475-3029-9

ISSN : 1157-996X

Référence électronique

Isabelle Chopin, "Le Traité d'Amsterdam : enjeux et défis en matière d'égalité de traitement 》, Cultures \& Conflits [En ligne], 45 | printemps 2002, mis en ligne le 22 mars 2006, consulté le 30 mars 2021. URL : http://journals.openedition.org/conflits/765 ; DOI : https://doi.org/10.4000/conflits.765

Ce document a été généré automatiquement le 30 mars 2021.

Creative Commons License 


\title{
Le Traité d'Amsterdam : enjeux et défis en matière d'égalité de traitement
}

The Amsterdam Treaty: Issues and Challenges concerning Equal Trea

\author{
Isabelle Chopin
}

1 La politique européenne en matière d'égalité de traitement en est à ses premiers pas ${ }^{1}$. Effectivement, jusqu'à présent des politiques et législations ont été adoptées considérant l'égalité de traitement entre hommes et femmes ou entre les ressortissants des pays de l'Union européenne, la situation des ressortissants de pays tiers étant réglementée au niveau européen par des accords d'association avec les pays d'origine. De plus, les domaines de l'immigration et de l'asile ne ressortaient pas de la compétence européenne. Le Traité d'Amsterdam pose de nouveaux défis en la matière. Ces nouveaux défis sont le fruit d'années de travail et de discussion et n'auraient pu voir le jour sans certaines étapes nécessaires.

2 La lutte contre le racisme, la xénophobie et les discriminations en découlant, et en faveur du principe d'égalité de traitement, a été initiée par les organisations non gouvernementales au niveau européen et national. En 1992, une coalition d'organisations non gouvernementales, fonctionnant en réseau informel, appelée le Groupe Ligne de Départ/Starting Line Group, convaincue de l'urgence et de la nécessité d'adopter une législation communautaire en la matière, présentait une proposition concrète de directive visant à lutter contre les discriminations fondées sur l'origine raciale ou ethnique. Depuis le début des années 1990, les institutions européennes ont adopté plusieurs résolutions communes concernant le racisme, soulignant la montée du phénomène et les dangers qu'il représente. Le Parlement européen, très sensible à ce sujet, a également adopté de nombreuses résolutions et rapports appelant la Commission européenne à œuvrer pour proposer des solutions pouvant remédier à l'inquiétante montée du racisme dans les Etats membres, notamment une législation calquée sur celle proposée par le Groupe Ligne de Départ. Cette proposition de directive très vite soumise aux différentes institutions européennes, basée sur l'ancien article 
235 (actuel article 308), s'est vue dénier toute possibilité d'existence en raison du manque de base légale dans le traité donnant compétence aux institutions européennes pour agir. Durant la préparation de la Conférence Intergouvernementale et pendant son déroulement, le Groupe Ligne de Départ, avec son réseau et d'autres organisations, a alors lancé une vaste campagne aux niveaux européen et nationaux afin de voir le traité amendé et d'y voir inscrite une clause de non-discrimination établissant clairement la compétence européenne dans ce domaine.

Il a cependant fallu attendre la forte médiatisation autour de dramatiques événements comme la profanation de cimetières juifs en France ou les incendies de maisons d'étrangers en Allemagne pour qu'à la suite d'une initiative franco-allemande, un premier instrument soit mis en place: la Commission Consultative Racisme et Xénophobie. Cette Commission, présidée par le Français Jean Kahn, avait alors pour but d'élaborer une stratégie à long terme afin de lutter de manière efficace contre le racisme et la xénophobie. Son rapport final comprenait différentes recommandations dont la nécessité d'amender le traité afin d'y inclure une clause de non-discrimination ainsi que la création de l'observatoire européen des phénomènes racistes et xénophobes. Cet Observatoire est actuellement situé à Vienne.

La Commission européenne est face à un climat politique favorable pour agir. Les organisations non gouvernementales ont réalisé un énorme travail d'information, de sensibilisation et de pression par le biais de campagnes et de propositions concrètes et ont réussi à faire inscrire le sujet sur l'agenda politique. Les Etats membres touchés par le phénomène semblent plus réceptifs et la Commission elle-même semble animée d'une volonté nouvelle et constructive. Le Commissaire aux Affaires Sociales de l'époque, l'irlandais Padraig Flynn, s'est largement impliqué afin que de nouvelles politiques et législations voient le jour et que la Commission présente des propositions constructives.

5 En 1995, la Commission européenne publie sa première communication contre le racisme. Cette communication reprend les activités menées au niveau européen et propose des domaines d'action future. L'approche utilisée consiste à incorporer la lutte contre le racisme dans les politiques déjà existantes tout en encourageant de nouvelles initiatives plus spécifiques. L’année 1997, Année européenne contre le Racisme, installe un premier cadre européen de lutte contre le racisme. Il s'agit là d'une reconnaissance officielle du problème et de la volonté d'y remédier. L'Année européenne contre le Racisme a contribué à créer une dynamique, à mettre en place de nouveaux partenariats, à sensibiliser au problème du racisme, à proposer des solutions et, enfin et surtout, à confirmer et consolider le rôle des institutions européennes en la matière.

6 En 1998, un Plan d'action est adopté qui vise à renforcer les partenariats avec les Etats membres, les organisations non gouvernementales, les partenaires sociaux, les autorités locales, les médias et les instances sportives. Ce plan d'action comprend 4 volets : l'ouverture aux initiatives législatives, l'intégration de la lutte contre le racisme dans les politiques et programmes de la Communauté, l'élaboration et l'échange de nouveaux modèles et le renforcement du travail d'information et de communication.

7 Parallèlement, lors de la conclusion de la Conférence Intergouvernementale, au sommet d'Amsterdam, l'article 13 a été adopté qui stipule que "Sans préjudice des autres dispositions du présent traité et dans les limites des compétences que celui-ci confêre à la communauté, le Conseil, statuant à l'unanimité sur proposition de la Commission et après consultation du Parlement européen, peut prendre les mesures nécessaires en vue de combattre 
toute discrimination fondée sur le sexe, la race ou l'origine ethnique, la religion ou les convictions, un handicap, l'âge ou l'orientation sexuelle». Sur la base de ce nouvel article, le développement du plan d'action a donné lieu à la présentation par la Commission européenne en novembre 1999 d'un set de trois propositions communément appelé « le paquet article $13 »$.

8 Le premier défi consistant en la prise en compte au niveau politique et législatif de la discrimination raciale et ethnique s'est vu matérialisé avec l'adoption de l'article 13. L'enjeu qui en découle est l'utilisation effective de cet article confirmant la mise en place d'une réelle volonté politique de combattre le racisme et la xénophobie.

Le "paquet article 13 » prend la forme de deux propositions de directives et d'un programme d'action.

* Le programme d'action qui est actuellement encore en discussion au Parlement européen et au Conseil, vise à soutenir les efforts des Etats membres dans l'élaboration de leurs législation et politique de lutte contre les discriminations notamment en mobilisant les acteurs clés et en favorisant l'échange d'information et de bonnes pratiques.

11 * La première proposition de directive est relative à la mise en œuvre du principe d'égalité de traitement entre les personnes sans distinction de race ou d'origine ethnique. Cette directive a été adoptée par le Conseil le 29 juin 2000. Elle est fortement inspirée de la proposition du Groupe Ligne de Départ, retravaillée après l'adoption de l'article 13 et de la législation relative aux discriminations fondées sur le sexe notamment la directive 97/80 sur le renversement de la charge de la preuve dans les cas de discrimination fondée sur le sexe. Elle prohibe et définit la discrimination directe et indirecte ainsi que le harcèlement. L'instruction de discriminer et les représailles constituent également une discrimination. La directive s'applique aux secteurs privé et public ainsi qu'à toute personne présente sur le territoire de l'Union européenne et donc également aux ressortissants de pays tiers. Cette interdiction de discriminer ne couvre pas, ainsi qu'il est mentionné dans la directive, la différence de traitement basée sur la nationalité. Le champ d'application ne se limite pas à l'emploi et comprend bien d'autres domaines de la vie quotidienne. La directive couvre l'emploi salarié ou indépendant (incluant le recrutement et les promotions), l'accès à la formation, les conditions de travail, l'appartenance et l'activité dans une organisation de travailleurs ou d'employeurs, la protection sociale, les avantages sociaux, l'éducation, la fourniture de biens et de services y compris le logement. Y est incluse la possibilité de recourir (sans obligation) ou de maintenir les actions positives déjà en place. Les droits de la défense y sont explicitement mentionnés ainsi que la possibilité pour les organisations ayant un intérêt légitime d'ester en justice. Il existe de la part des Etats membres un devoir d'informer et de favoriser le dialogue social et avec les organisations non gouvernementales. De plus, les Etats membres devront désigner un organe qui apportera assistance aux victimes, fera des recherches et publiera des rapports indépendants. Une justification de la différence de traitement est possible lorsque la nature d'une activité spécifique requiert cette différence de traitement et la directive impose le déplacement de la charge de la preuve. Parmi les objectifs de la communauté des organisations non gouvernementales qui n'ont pas été atteints, figure la prise en compte de la discrimination religieuse. Aucun compromis politique n'ayant été possible avec certaines églises. 
12 * La deuxième proposition de directive porte sur la création d'un cadre général en faveur de l'égalité de traitement en matière d'emploi et de travail et concerne tous les motifs de discrimination listés dans l'article 13 (les cas de discriminations, fondés sur l'origine raciale ou ethnique, seront supprimés de cette deuxième directive afin d'éviter tout chevauchement avec la directive adoptée en juin 2000). Cette proposition de directive est très similaire à la première mais beaucoup plus réduite au niveau de son champ d'application puisqu'elle se limite aux domaines suivants : les conditions d'accès à l'emploi salarié, à une activité ou profession non salariée ; l'accès à tous les types et à tous les niveaux d'orientation professionnelle, de formation professionnelle, de perfectionnement et de formation, de reconversion; les conditions de licenciement et de rémunération; et l'affiliation à et l'engagement dans une organisation de travailleurs ou d'employeurs ou toute autre organisation. Cette directive est actuellement en discussion devant le Parlement européen et le Conseil.

13 Les enjeux soulevés par le traité d'Amsterdam dans ce domaine sont en phase de réalisation puisque la problématique de l'égalité de traitement a été soulevée et prise en compte au niveau politique par le biais de propositions concrètes. Cependant, ainsi que le clament de façon répétée et continue les organisations non gouvernementales actives dans le domaine, l'adoption de la directive visant à lutter contre les discriminations basées sur l'origine raciale ou ethnique a fait ressortir la difficulté de lutter pour la mise en œuvre du principe d'égalité de traitement sans prendre en compte les discriminations fondées sur la nationalité. L'adoption du traité d'Amsterdam ouvre à cet égard une brèche. Effectivement, un nouveau titre IV sur les politiques en matière de « visas, d'asile, d'immigration et autres politiques relatives à la libre circulation des personnes » a été ajouté au traité. Ce transfert de compétences du 3ème pilier (coopération intergouvernementale) au 1er pilier (compétence communautaire) constitue une énorme avancée dans l'élaboration de politiques communes dans les domaines de l'immigration et de l'asile, et permet également un renforcement démocratique puisque le Parlement européen devra être consulté et que la Cour de Justice européenne verra son rôle renforcé avec un pouvoir d'interprétation sur demande des tribunaux de dernière instance. Il est cependant à noter que ce transfert de compétences n'interviendra de manière effective qu'après une période transitionnelle de 5 ans (à compter du 1er mai 1999, date d'entrée en vigueur du traité) et que certaines limitations (prise de décision à l'unanimité, droit de consultation réduit du Parlement européen et l'impossibilité pour la Cour de Justice européenne d'émettre des décisions sur des questions d'interprétation aux tribunaux nationaux de moindre instance) prévues pour cette période de transition pourront être maintenues. Une nouvelle décision du Conseil devra déterminer le statut de ces limitations après la période de transition.

14 Le nouveau défi se situe à deux niveaux: assurer la mise en place de nouvelles politiques équitables par le biais de propositions de qualité durant la période de transition et faire lever les limitations mentionnées ci-dessus après cette période de cinq ans. La Commission européenne travaille déjà sur différentes propositions de directives. Le Migration Policy Group (MPG) et l'Immigration Law Practioners' Association (ILPA) ont collaboré sur le sujet et publié conjointement 6 propositions de directives concernant l'asile, les visas et le contrôle aux frontières, le droit au regroupement familial, l'admission des migrants, les résidents permanents et les migrants irréguliers. Ces propositions doivent servir de base à la discussion, provoquer le débat aux niveaux 
européen et nationaux et apporter leur expertise à l'élaboration de ces nouvelles politiques.

Les négociations du traité d'Amsterdam ainsi que son adoption ont révélé un profond changement dans la manière d'élaborer de nouvelles politiques. De nouveaux acteurs, comme les organisations non gouvernementales, ont été reconnus et pris en considération sur la scène européenne. Le travail effectué par ces organisations a porté ses fruits puisqu'elles ont réussi à faire inscrire et à maintenir leurs préoccupations sur l'agenda politique. L'exemple de la directive adoptée dernièrement sur les discriminations raciales et ethniques en est un exemple criant, même si l'adoption de cette directive a probablement été accélérée en raison des résultats des dernières élections en Autriche. Les interlocuteurs en termes d'élaboration de nouvelles politiques ne sont plus seulement les gouvernements nationaux avec leur cohorte d'experts, d'administrateurs et de politiciens mais également les représentants de la société civile et des victimes. Des processus de consultation avec les organisations non gouvernementales ont eu lieu au niveau du Parlement européen, de la Commission européenne et dans certains Etats membres. Ces consultations parfois maladroites et souvent décriées ont cependant le mérite d'exister alors que le procédé était impensable il y a peu. D'aucuns penseront que ces consultations sont un alibi tentant de légitimer certaines décisions, ce qui peut être vrai. Libre aux organisations consultées d'occuper le peu de place qui leur est octroyée et de faire entendre leurs revendications comme une nouvelle interprétation de l'article 12 traitant de la discrimination sur base de la nationalité - considéré jusqu'ici comme ne s'appliquait qu'aux ressortissants de l'Union européenne. Il est évident que la problématique de la citoyenneté va de nouveau être soulevée, logiquement inscrite dans un processus d'intégration des ressortissants de pays tiers, de leur participation pleine et entière à la vie de leur pays de résidence et de révision d'un concept parfois restrictif, désuet et conservateur de la notion de citoyenneté.

Les enjeux politiques sont énormes dans le domaine de l'égalité de traitement et des politiques d'immigration et d'asile. La volonté de parvenir à des politiques plus égalitaires ne pourra se réaliser sans une prise de conscience globale, une prise de responsabilité partagée des acteurs clés et des décideurs et sans une vision à long terme du devenir de l'Union européenne, de ses citoyens et de ses relations avec les autres Etats, voisins ou plus éloignés, industrialisés ou plus pauvres. Ces nouveaux enjeux politiques vont donner lieu à de sévères négociations dans lesquelles tous ces nouveaux interlocuteurs ont une place à prendre, une chance à saisir et un rôle à jouer.

\section{NOTES}

1. Conférence prononcée en juin 2000 dans le cadre d'un séminaire organisé par le CERI et le Centre d'Etudes sur les Conflits. 


\section{RÉSUMÉS}

Several organizations and agreements have produced policies concerning equal treatment of races and nationalities in Europe. The 2000 Amsterdam Summit proposed a list of groups to protect from discrimination, a plan for opposing discrimination in the $\mathrm{EU}$, a directive for instituting equal public and private treatment of racial and ethnic groups, as well as another directive protecting all the listed groups from workplace discrimination. Implementation of the new policies will affect the EU's structure ; a 5-year transitional period is planned. The inclusion of nongovernmental organizations in the anti-discrimination dialogue is commended. An appended excerpt from the European Council's official journal (19 July 2000) includes the directive on racial and ethnic discrimination.

INDEX

Index géographique : Union européenne

Mots-clés : construction européenne, droit, droit européen, genre

Thèmes : Traité de l'Union européenne (TUE) 